\title{
Extensive lipoma-like changes of myxoid liposarcoma: morphologic, immunohistochemical, and molecular cytogenetic analyses
}

\author{
Hiroshi Iwasaki • Masako Ishiguro • Jun Nishio • \\ Mikiko Aoki • Ryohei Yokoyama • Koichiro Yokoyama • \\ Kenichi Taguchi • Kazuki Nabeshima
}

Received: 2 November 2014 /Revised: 2 January 2015 / Accepted: 19 January 2015 / Published online: 4 February 2015

(C) The Author(s) 2015. This article is published with open access at Springerlink.com

\begin{abstract}
Myxoid liposarcomas (MLSs) with extensive lipoma-like changes (MLSLC) are rare, and it is often difficult to distinguish them from well-differentiated liposarcoma (LS)/ dedifferentiated LS (WDLS/DDLS) with myxoid changes. For the characterization of these neoplasms, we studied 8 MLSLCs, 11 ordinary MLSs, 4 WDLSs, and 6 DDLSs. Cytogenetically, MLSLC and ordinary MLS were characterized by $\mathrm{t}(12 ; 16)(\mathrm{q} 13 ; \mathrm{p} 11)$ and FUS-DDIT3 fusion gene, whereas WDLS/DDLS lacked the fusion gene but possessed giant marker/ring chromosomes. Both lipoma-like and myxoid components of the same MLSLC exhibited the identical FUS-DDIT3, as confirmed by fluorescence in situ hybridization (FISH) and reverse transcription polymerase chain reaction (RT-PCR). Immunohistochemically, MDM2 and CDK4 were positive in WDLS/DDLS but negative in MLSLC and ordinary MLS. PPAR $\gamma, \mathrm{C} / \mathrm{EBP} \alpha$, adipophilin, and perilipin were found in each type of LS. Adipophilin was expressed chiefly in tiny fat droplets of immature lipoblasts, whereas perilipin showed a strong positive staining in large fat
\end{abstract}

H. Iwasaki $(\bowtie) \cdot$ M. Ishiguro $\cdot$ M. Aoki $\cdot$ K. Nabeshima

Department of Pathology, Fukuoka University School of Medicine,

7-45-1 Nanakuma, Jonan-ku, Fukuoka 814-0180, Japan

e-mail: iwasaki@fukuoka-u.ac.jp

J. Nishio

Department of Orthopedic Surgery, Fukuoka University School of Medicine, 7-45-1 Nanakuma, Jonan-ku, Fukuoka 814-0180, Japan

R. Yokoyama $\cdot$ K. Yokoyama

Department of Orthopedic Surgery, National Hospital Organization

Kyushu Cancer Center, 3-1-1 Notame,

Minami-ku, Fukuoka 811-1395, Japan

K. Taguchi

Department of Pathology, National Hospital Organization Kyushu Cancer Center, 3-1-1 Notame, Minami-ku, Fukuoka 811-1395, Japan vacuoles of signet ring and multivacuolated lipoblasts. The Ki-67 labeling index was lower in the lipoma-like component of MLSLC when compared with the myxoid component of the same tumors as well as ordinary MLS $(p<0.001)$. When compared with ordinary MLS, MLSLC may be less aggressive in clinical behavior (rare recurrences or metastases) after a wide surgical excision. In conclusion, the distinction between MLSLC and WDLS/DDLS is important, because of the differences of molecular cytogenetic features as well as clinical behaviors between these distinct sarcomas presenting similar morphologic features. In addition, the combined immunohistochemical detection of adipophilin and perilipin may provide a useful ancillary tool for identification of lipoblastic cells in soft tissue sarcomas.

Keywords Myxoid liposarcoma · Dedifferentiated liposarcoma · Lipoma-like liposarcoma $\cdot$ FUS-DDIT3 . Fusion gene

\section{Introduction}

Myxoid liposarcoma (MLS) is the second most common type of liposarcoma (LS) after atypical lipomatous tumor/welldifferentiated LS (ALT/WDLS) [1, 2]. MLS usually presents as a large painless mass within the deep soft tissue of the extremities in young adults and middle-aged individuals. Histologically, MLS is composed of predominantly uniform round- to oval-shaped primitive mesenchymal cells admixed with signet ring or multivacuolated lipoblasts in a prominent myxoid stroma, which is rich in a delicate arborizing "chicken-wire" capillary vasculature. Included in this category are lesions formerly known as round cell LS (RCLS) 
characterized by hypercellular round cell morphology, which is associated with a significantly poor prognosis [1-3].

Although a variable number of signet ring lipoblasts are found in MLS, extensive mature adipocytic differentiation forming a lipoma-like component is very rare [4-6]. Morphologically, it is often difficult to distinguish MLS with extensive lipoma-like changes (MLSLC) from ALT/WDLS with myxoid changes, but the severe degree of nuclear atypia may help to exclude MLS/MLSLC [7]. Cytogenetically, most cases of MLS show specific chromosomal translocation $\mathrm{t}(12 ; 16)(\mathrm{q} 13 ; \mathrm{p} 11)$ resulting in the formation of a FUS-DDIT3 (also known as TLS-CHOP) fusion transcript [8-17], although occasional tumors exhibit the variant $\mathrm{t}(12 ; 22)$ (q13;q12), producing an EWSR1-DDIT3 fusion transcript $[1,18]$. On the other hand, ALT/WDLS is characterized by supernumerary ring chromosome and/or giant markers with amplification of the $12 \mathrm{q} 13 \sim \mathrm{q} 15$ region, which is often associated with overexpression of CDK4 and MDM2 [1, 17, 19-23].

Myxoid areas within ALT/WDLS and dedifferentiated LS (DDLS) sometimes possess a prominent plexiform vascularity with thin-walled arborizing capillaries, creating a resemblance to MLS especially when interspersed small fat cells are present $[7,24-26]$. On the basis of molecular and immunohistochemical studies, de Vreeze et al. [24] suggested that apparent primary retroperitoneal MLS/RCLS could be recognized as WDLS/DDLS with morphological features mimicking MLS/ RCLS. They considered that finding of the MLS-specific translocations (fusion genes) in a retroperitoneal LS is highly suggestive of metastasis and should prompt search for a primary lesion outside the retroperitoneum.

In addition, rare cases of mixed-type LS composed of an admixture of MLS and WDLS have been reported by some investigators [17, 27]. More recently, Deyrup et al. [28] reported a new group of "fibrosarcoma-like lipomatous neoplasm," which were composed of low-grade spindle cells showing varying degrees of lipoblastic differentiation and sometimes accompanied by abundant myxoid stoma and thin-walled arborizing capillaries similar to those of MLS, but these tumors lacked molecular cytogenetic characteristics of other types of lipomatous tumors.

The development of normal fat cells is considered to be regulated by various factors including peroxisome proliferator-activated receptor- $\gamma$ (PPAR $\gamma)$ and CCAAT/enhancer-binding protein- $\alpha(\mathrm{C} / \mathrm{EBP} \alpha)$ [29]. PPAR $\gamma$ may regulate the expression of lipid droplet-associated proteins including adipophilin and perilipin in normal tissues [30,31], but little is known about the mechanism of adipocytic differentiation in MLSLC.

In order to clarify the true nature of rare lipomatous differentiation in MLS, we studied eight cases of MLSLC, by using immunohistochemistry (MDM2, CDK4, PPAR $\gamma, \mathrm{C} / \mathrm{EBP} \alpha$, adipophilin, perilipin, and $\mathrm{Ki}-67$ ), chromosome analysis, fluorescence in situ hybridization (FISH), and reverse transcription polymerase chain reaction (RT-PCR). In addition, ordinary MLS (11 cases), WDLS (4 cases), and DDLS (6 cases) were studied as controls. To our knowledge, this is the first report describing the detailed molecular cytogenetic and clinicopathologic features of MLSLC.

\section{Materials and methods}

\section{Tumor material and patient data}

The consultation and archival files of molecular cytogenetic analysis of soft tissue tumors in the Department of Pathology, Fukuoka University School of Medicine, between 1987 and 2012 were searched for MLS with or without welldifferentiated lipoma-like components. Eight cases of MLSLC were selected for the present study. In addition, 11 cases of ordinary MLS without lipoma-like components, 4 cases of WDLS, and 6 cases of dedifferentiated LS (DDLS) with myxoid changes were studied as controls.

Clinical parameters, including gender, age, location, and macroscopic features, were obtained from medical records. In all cases, histologic sections were available for pathologic review, and the diagnosis was confirmed according to the WHO (2013) classification. Formalin-fixed and paraffinembedded tumor tissues available in each case were used for immunostaining and molecular analysis. In 16 cases, fresh tumor tissues were utilized for chromosomal analysis as well as molecular study. Follow-up information was obtained from the referring clinicians and from the existing medical records in accord with institutional guidelines.

\section{Immunohistochemical staining}

For immunohistochemistry, 3- $\mu$ m-thick paraffin-embedded tissue sections were mounted on silane-coated glass slides, deparaffinized, and heated in antigen retrieval buffer using a pressure cooker for $10 \mathrm{~min}$ or a microwave for $30 \mathrm{~min}$. The following primary antibodies were used: MDM2 (dilution 1/100; Calbiochem, Darmstadt, Germany), CDK4 (dilution 1/200; Invitrogen, Camarillo, CA), PPAR $\gamma$ (dilution 1/100; Perseus Proteomics, Tokyo, Japan), C/EBP $\alpha$ (dilution 1/200; Cell Signaling Technology, Danvers, MA), adipophilin (dilution 1/20; Fitzgerald, Acton, MA), perilipin (dilution 1/200; Cell Signaling Technology, Danvers, MA), and Ki-67 (clone MIB-1, dilution 1/200; Dako, Glostrup, Denmark). Immunohistochemical staining was performed by using the Nichirei Histofine Simple Stain MAX PO (MULTI) (Nichirei Biosciences Inc., Tokyo, Japan). The reactions were visualized with diaminobenzidine, and the sections were counterstained with Mayer's hematoxylin. For MDM2, CDK4, PPAR $\gamma, \mathrm{C} / \mathrm{EBP} \alpha$, adipophilin, and perilipin, the immunoreactivity was graded semiquantitatively as negative $(0 \%), 1+(<25 \%$ of neoplastic 
cells reactive), $2+$ ( 25 to $50 \%$ of neoplastic cells reactive), and $3+(>50 \%$ of neoplastic cells reactive). The Ki-67 labeling index was obtained as a percentage of positive nuclei by counting 500 neoplastic cells within the areas exhibiting the highest labeling index (hot spots). The Mann-Whitney $U$ test was used to compare the differences of the Ki-67 index.

Cytogenetic analysis

Primary cell cultures, harvesting, and preparation of slides were carried out as previously described [9, 21, 22]. Chromosome analysis was performed on GTG-banded (Giemsa/trypsin) metaphases, and the karyotypes were described according to the International System for Human Cytogenetic Nomenclature [32].

FISH analysis for DDIT3 break apart

FISH was performed on paraffin-embedded tissue sections (cases $1-8,12,13,17,18,21-25$ ), by using a commercially available DDIT3 (CHOP) (12q13) Dual Color, Break Apart Rearrangement Probe (Abbott Molecular Inc.). Briefly, 4- $\mu$ mthick paraffin-embedded tissue sections were deparaffinized, dehydrated, and incubated with protease and pretreated by the Vysis Paraffin Pretreatment IV \& Post-Hybridization Wash Buffer Kit (Abbott Molecular Inc.) according to the manufacturer's instructions $[4,33]$. The probe and slides were codenatured at $80^{\circ} \mathrm{C}$ for $5 \mathrm{~min}$ and incubated at $37^{\circ} \mathrm{C}$ overnight in a humidified chamber. Posthybridization washing was performed following standard procedures, and the nuclei were counterstained with DAPI. The slides were examined using a fluorescence microscope with appropriate excitation and emission filters. At least 100 morphologically intact, nonoverlapping nuclei of tumor cells from either myxoid or lipomatous areas were counted. FISH was considered rearranged if more than $10 \%$ of nuclei showed break apart of the dual-color probe signal for the targeted locus.

\section{RT-PCR for FUS-DDIT3}

RNA was extracted from fresh-frozen tumor tissues (cases 1, $2,6-12,14-21,23-25,27-29)$ as well as formalin-fixed and paraffin-embedded tumor samples (cases 1, 3-8, 13, 22, 26) by using TRIzol ${ }^{\circledR}$ reagent (Life Technologies Japan, Tokyo, Japan) according to the manufacturer's recommendations. In seven cases of MLSLC (cases 1, 3-8), the paraffin sections were dissected under a microscope, and the samples were taken separately from myxoid and lipoma-like components, which were simultaneously identified by examining the adjacent serial sections stained with $\mathrm{H} \& \mathrm{E}$.

RNA was reverse transcribed into complementary DNA (cDNA) by PrimScript ${ }^{\circledR}$ II first-strand cDNA Synthesis Kit (Takara Bio, Tokyo, Japan). The PCR to amplify a cDNA that corresponds to FUS-DDIT3 fusion gene was performed by using
KOD-Plus-Ver. 2 (Toyobo, Tokyo, Japan) with the FUS ex 5 and DDIT3 primer set amplifying a 160-bp (FUS-DDIT3 type II) fragment and a 436-bp (FUS-DDIT3 type I) fragment or the FUS ex 7 and DDIT3 primer set amplifying a 129-bp (FUSDDIT3 type I) fragment, according to Powers et al. [34]. PCR products were analyzed by Microchip Electrophoresis System for DNA/RNA Analysis with DNA-1000 Kit (Shimadzu, Tokyo, Japan).

\section{Results}

Clinical features

Clinical information of 29 patients examined is summarized in Table 1. One of five MLSLCs and four of nine ordinary MLSs with follow-up data showed local recurrences. As for WDLS and DDLS, all six retroperitoneal tumors recurred after surgery, whereas four tumors arising in the extremities exhibited no recurrences after a complete wide excision. A remote metastasis was found in one MLSLC, two ordinary MLSs, and one DDLS. One patient with retroperitoneal DDLS was dead of disease

\section{Macroscopic features}

Grossly, MLSs were multinodular or lobulated and often involved the skeletal muscle. The size of tumor was described in six cases of MLSLC, ranging from 7 to $13 \mathrm{~cm}$ in the greatest dimension (mean, $9.5 \mathrm{~cm}$; median, $9.5 \mathrm{~cm}$ ). All eight tumors of MLSLC contained lipoma-like yellowish areas in varying proportions (30-90\%), admixed with myxoid areas resembling ordinary MLS. Foci of necrosis and hemorrhage were found in the large tumors (cases 5 and 8). The myxoid areas of MLSLC and ordinary MLS were typically gelatinous, jellylike with an abundant myxoid substance.

WDLSs were characterized by large yellowish masses lobulated by fibrous septa. Foci of sclerosis and myxoid changes were often observed. DDLS generally exhibited yellowish lipoma-like components, coexisting with solid gray-whitish areas corresponding to the dedifferentiation. Proportion of lipoma-like to dedifferentiated components varied from tumor to tumor, and one DDLS was composed almost completely of a dedifferentiated component (case 29).

Histologic features

All eight tumors of MLSLC contained lipoma-like well-differentiated component comprising 30-90\% (mean $49 \%$ ) of the mass (Table 1, Figs. 1 and 2a, b), whereas the remaining 11 control cases of ordinary MLS lacked distinct lipoma-like components. The lipoma-like components of MLSLC showed abundant mature adipocytes with considerable variation in cellular size (Figs. 1 and 2a), bearing superficial resemblance 
Table 1 Clinicopathologic features of liposarcomas with or without lipoma-like component

\begin{tabular}{|c|c|c|c|c|c|c|c|c|c|c|}
\hline Case & Age (year) & Sex & Site & Histologic type & $\begin{array}{l}\text { Lipoma-like } \\
\text { component (\%) }\end{array}$ & Therapy & $\begin{array}{l}\text { Follow-up } \\
\text { time (month) }\end{array}$ & Recurrence & Metastasis & Outcome \\
\hline 1 & 23 & $\mathrm{~F}$ & rt. lower leg & MLSLC & 40 & Wide excision & 66 & No & No & ANED \\
\hline 2 & 66 & M & rt. lower leg & MLSLC & 40 & Wide excision & 25 & Yes, 1 & Yes, 1 & ANED \\
\hline 3 & 75 & M & 1t. thigh & MLSLC & 50 & Excision & NA & & & LTF \\
\hline 4 & 35 & $\mathrm{~F}$ & rt. lower leg & MLSLC & 30 & Excision & NA & & & LTF \\
\hline 5 & 74 & M & 1t. thigh & MLSLC & 90 & Excision & NA & & & LTF \\
\hline 6 & 66 & $\mathrm{~F}$ & 1t. thigh & MLSLC & 30 & Wide excision & 27 & No & No & ANED \\
\hline 7 & 66 & M & rt. groin & MLSLC & 70 & Wide excision & 23 & No & No & ANED \\
\hline 8 & 51 & M & rt. thigh & MLSLC & 40 & Wide excision & 8 & No & No & ANED \\
\hline 9 & 28 & M & Mediastinum & MLS & 0 & Excision & 60 & Yes, 1 & Yes, 1 & AWD \\
\hline 10 & 39 & $\mathrm{~F}$ & 1t. poplitea & MLS & 0 & Wide excision & 71 & No & No & ANED \\
\hline 11 & 39 & $\mathrm{~F}$ & 1t. thigh & MLS & 0 & Excision & NA & & & LTF \\
\hline 12 & 35 & M & 1t. thigh & MLS & 0 & Wide excision & 75 & Yes, 3 & No & ANED \\
\hline 13 & 52 & $\mathrm{~F}$ & 1t. thigh & MLS & 0 & Wide excision & 62 & Yes, 1 & No & ANED \\
\hline 14 & 54 & M & Retroperitoneum & MLS & 0 & Wide excision & 8 & Yes, 2 & Yes, 1 & AWD \\
\hline 15 & 44 & $\mathrm{~F}$ & Thoracic wall & MLS & 0 & Wide excision & NA & & & LTF \\
\hline 16 & 48 & M & 1t. knee & MLS & 0 & Wide excision & 22 & No & No & ANED \\
\hline 17 & 53 & $\mathrm{~F}$ & lt. lower leg & MLS & 0 & Wide excision & 52 & No & No & ANED \\
\hline 18 & 36 & M & r. buttock & MLS & 0 & Wide excision & 54 & No & No & ANED \\
\hline 19 & 36 & $\mathrm{~F}$ & rt. ankle & MLS & 0 & Wide excision & 51 & No & No & ANED \\
\hline 20 & 69 & M & rt. thigh & WDLS & 80 & Wide excision & 49 & No & No & ANED \\
\hline 21 & 48 & $\mathrm{~F}$ & Retroperitoneum & WDLS & 70 & Excision & 69 & Yes, 3 & No & AWD \\
\hline 22 & 67 & $\mathrm{~F}$ & Retroperitoneum & WDLS & 95 & Excision & NA & Yes, 1 & & LTF \\
\hline 23 & 74 & $\mathrm{~F}$ & 1t. thigh & WDLS & 98 & Wide excision & 23 & No & No & ANED \\
\hline 24 & 50 & $\mathrm{~F}$ & Retroperitoneum & DDLS & 40 & Excision & 104 & Yes, 3 & Yes & AWD \\
\hline 25 & 61 & M & Retroperitoneum & DDLS & 20 & Excision & 7 & Yes, 1 & No & AWD \\
\hline 26 & 73 & $\mathrm{~F}$ & Retroperitoneum & DDLS & 70 & Incomplete Excision & 138 & Yes, 1 & No & DOD \\
\hline 27 & 58 & $\mathrm{~F}$ & Retroperitoneum & DDLS & 10 & Excision & 25 & Yes, 1 & No & ANED \\
\hline 28 & 83 & $\mathrm{~F}$ & rt. thigh & DDLS & 5 & Wide excision & 33 & No & No & ANED \\
\hline 29 & 79 & $\mathrm{~F}$ & 1t. lower leg & DDLS & 0 & Wide excision & 9 & No & No & ANED \\
\hline
\end{tabular}

$A N E D$ alive and no evidence of disease, $A W D$ alive with disease, $D D L S$ dedifferentiated liposarcoma, $D O D$ dead of disease, $L T F$ lost to follow-up, $M L S$ myxoid liposarcoma, MLSLC MLS with lipoma-like component, $W D L S$ well-differentiated liposarcoma

to WDLS (Fig. 2e). In most tumors of MLSLC, there were gradual transitional zones (Fig. 2b) between the myxoid and lipoma-like components, but some tumor demonstrated focally sharp borders (Fig. 1) between the two components (cases 5 and 8). However, no significant difference of histologic features was found between the myxoid areas of MSLLC and ordinary MLS (Fig. 2c, d). The myxoid areas were made up of a proliferation of small round or stellate cells loosely floating in an abundant myxoid stroma, rich in delicate arborizing capillary blood vessels. Varying numbers of lipoblasts including univacuolated signet ring cells and some small multivacuolated cells were admixed with immature round or stellate cells, but typical spiderweb or giant lipoblasts with bizarre hyperchromatic nuclei were absent.

In all four cases of WDLS, tumors were composed of predominant mature adipocytes interspersed with varying number of atypical cells having hyperchromatic nuclei (Fig. 2e). Multivacuolated lipoblasts with spiderweb features were observed among the tumor cells. Ill-defined focal myxoid areas with spindle or stellate cells were commonly seen in WDLS. DDLS (six cases) contained well-defined nonlipogenic dedifferentiated areas (Fig. $2 \mathrm{f}-\mathrm{h}$ ) in addition to the lipoma-like well-differentiated areas. Both components were usually seen in the same DDLS, but one tumor was composed almost completely of a dedifferentiated component (case 29). The dedifferentiated areas consisted mainly of a high-grade pleomorphic/spindle cell component reminiscent of malignant fibrous histiocytoma (Fig. 2g), but varying degrees of myxoid changes (Fig. 2f, h) were observed in all cases of DDLS examined. In case 24, recurrent DDLS showed prominent cartilaginous metaplasia as well as a low-grade fibromatosis-like component. 


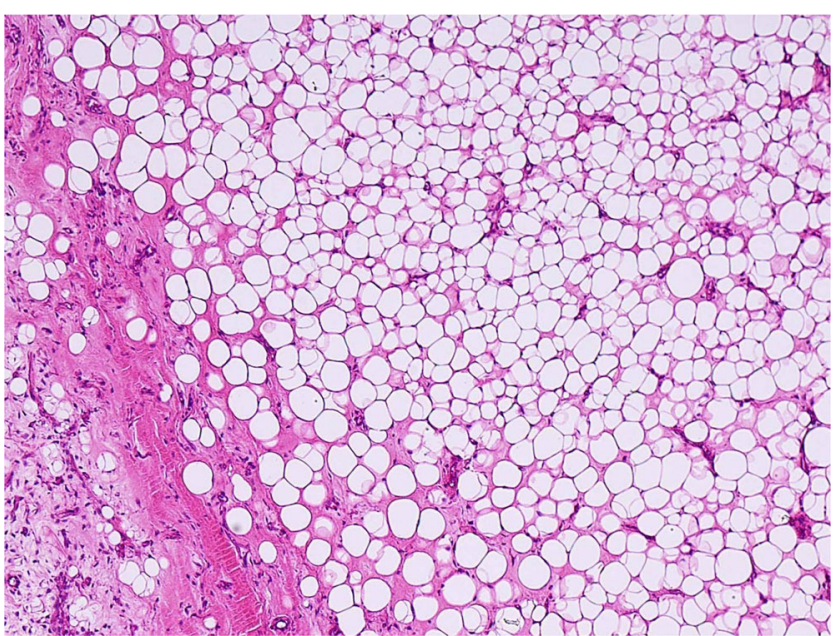

Fig. 1 Myxoid liposarcoma with extensive lipoma-like changes. The bulk of this tumor is composed of mature adipocytes with considerable variation in cellular size and shape, bearing superficial resemblance to atypical lipomatous tumor/well-differentiated liposarcoma. Notice a small amount of myxoid component with arborizing capillary blood vessels on the lower left corner

\section{Immunohistochemical findings}

In MLSLC, the tumor cells were uniformly negative for CDK4 (Fig. 3a) and MDM2 (Fig. 3b) in both lipoma-like and myxoid areas. Ordinary MLS showed essentially the same immunoreactivity as that of MLSLC. On the other hand, WDLS (Fig. 3c, d) and DDLS were variably positive for CDK4 and MDM2, presenting a sharp contrast to MLS with or without lipoma-like components.

$\operatorname{PPAR} \gamma$ and $\mathrm{C} / \mathrm{EBP} \alpha$ were positive in all tumors of each LS type (Table 2, Fig. 3e, f), but the cells expressing these proteins were somewhat more common in undifferentiated myxoid areas than in lipoma-like components. Adipophilin was expressed in each tumor of MLSLC (Fig. 3g) and ordinary MLS, although myxoid areas contained more positive cells than lipoma-like components did. Generally, reactivity for adipophilin was stronger in immature lipoblastic cells than in mature fat cells. WDLS and DDLS also showed varying positivity for adipophilin in tumor cells, but the numbers of positive cells were usually fewer, when compared with MLS with or without lipoma-like components. Perilipin was positive in all cases of MLSLC (Fig. 3h) and ordinary MLS. However, because the perilipin decollated large fat vacuoles in the cytoplasm, mature fat cells and signet ring lipoblasts as well as multivacuolated lipoblasts revealed a stronger reactivity, when compared with immature cells. In WDLS and DDLS, mature fat cells and spiderweb lipoblasts showed more intense reactivity for perilipin than undifferentiated round or spindle cells did.

The Ki-67 labeling index (Table 2, Fig. 3i, j) was significantly lower in the lipoma-like components of MLSLC (range, 0.8-3.6; mean, 2.7; median, 2.9) than either in the myxoid components of MLSLC (range, 7.4-20.2; mean, 12.2; median, 11.2) $(p<0.001)$ or in the ordinary MLS (range, 6.4-22.2; mean, 14.4; median, 15.6) ( $p<0.001)$. In addition, there was no significant difference of the $\mathrm{Ki}-67$ index between ordinary MLS and myxoid component of MLSLC.

\section{Chromosome analysis}

Chromosomal analysis with G-band technique was performed in three cases of MLSLC and in eight cases of ordinary MLS. All MLSLC tumors examined demonstrated $\mathrm{t}(12 ; 16)(\mathrm{q} 13 ; \mathrm{p} 11)$ characteristic of MLS (Table 2, Fig. 4a), although a few additional aberrations were found in some cases. Supernumerary ring chromosomes or giant marker chromosomes were never found in MLS with or without lipoma-like components. On the contrary, WDLS (three cases) and DDLS (one case) demonstrated supernumerary ring (Fig. 4b) and/or giant marker chromosomes. In addition, a complex karyotype was found in a case of DDLS.

\section{FISH for detecting break apart of DDIT3}

FISH was performed in all eight cases of MLSLC and in four cases of ordinary MLS. Break apart of DDIT3 was confirmed in each MLS regardless of lipoma-like changes (Table 2). In MLSLC, both lipoma-like and myxoid areas of the same histologic sections showed identical break-apart signals (Fig. 4c, d). The sarcoma cells with DDIT3 break apart exhibited one yellow (fusion signal), one green, and one red (break-apart signal pattern), whereas normal cells (inner control such as vascular endothelia cells) possessed a two-fusion signal pattern (yellow or red and green overlapping) representing two intact copies of the DDIT3 gene. On the other hand, WDLS (four cases) and DDLS (six cases) lacked break-apart signals of DDIT3. In three cases of DDLS, the break-apart signals were uncountable because of the presence of densely packed multiple signals.

\section{RT-PCR for FUS-DDIT3}

FUS-DDIT3 fusion transcript was found in all tumors of MLS examined, including 8 cases of MLSLC and 11 cases of ordinary MLS (Table 2). The incidence of FUS-DDIT3 fusion variants was almost the same in MLSLC [type I, two cases (25\%); type II, six cases (75\%)] and ordinary MLS [type I, three cases (27\%); type II, eight cases (73\%)]. In addition, both myxoid and lipoma-like components dissected from the same paraffin sections of MLSLC exhibited the identical fusion variant of FUS-DDIT3 (Fig. 4e). On the contrary, none of the WDLS/DDLS showed FUS-DDIT3 fusion. 
Fig. 2 a-c Myxoid liposarcoma (MLS) with lipoma-like changes (MLSLC). a Focal myxoid change in lipoma-like component closely resembling welldifferentiated liposarcoma. b Transitional zone between myxoid and lipoma-like areas. c Myxoid area of MLSLC with immature round cells and signet ring lipoblasts in an abundant myxoid stroma. d Ordinary MLS with arborizing capillary blood vessels. e Well-differentiated liposarcoma with focal myxoid changes showing similarity to MLSLC. f Dedifferentiated liposarcoma (DDLS) exhibits nonlipogenic components with myxoid changes. $\mathbf{g}$ Undifferentiated pleomorphic sarcoma-like area of DDLS. $\mathbf{h}$ Marked myxoid changes in DDLS with arborizing capillary blood vessels, reminiscent of MLS
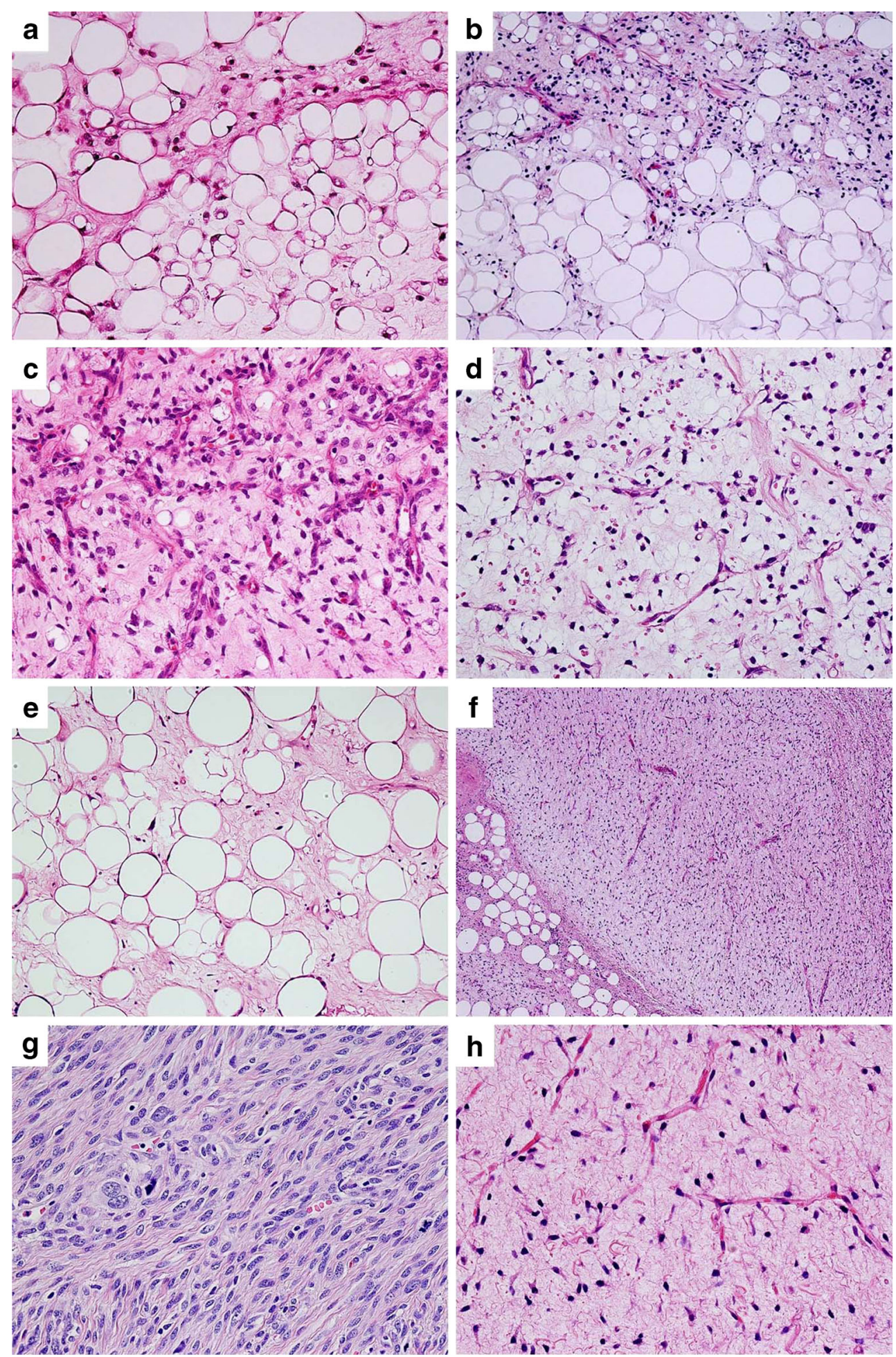

\section{Discussion}

LSs rarely exhibit unusual histologic features with combined patterns of lipoma-like WDLS and MLS [4, 6, 12, 23]. However, the distinction between MLSLC and WDLS/DDLS with myxoid changes is often difficult
Fig. 3 Immunostaining. a, b Myxoid liposarcoma with lipoma-like change (MLSLC) shows negative staining for CDK4 and MDM2. c, d Well-differentiated liposarcoma exhibit nuclear expressions of CDK 4 and MDM2. e, f MLSLC shows positive nuclear staining for PPAR $\gamma$ and $\mathrm{C} / \mathrm{EBP} \alpha$. g Adipophilin is strongly positive in immature lipoblastic cells of MLSLC. $\mathbf{h}$ Perilipin is positive in large fat vacuoles of mature adipocytes and multivacuolated lipoblasts of MLSLC. i, j The Ki-67 index is very low in $\mathbf{i}$ the lipoma-like area of MLSLC, when compared with $\mathbf{j}$ the myxoid area of the same tumor 

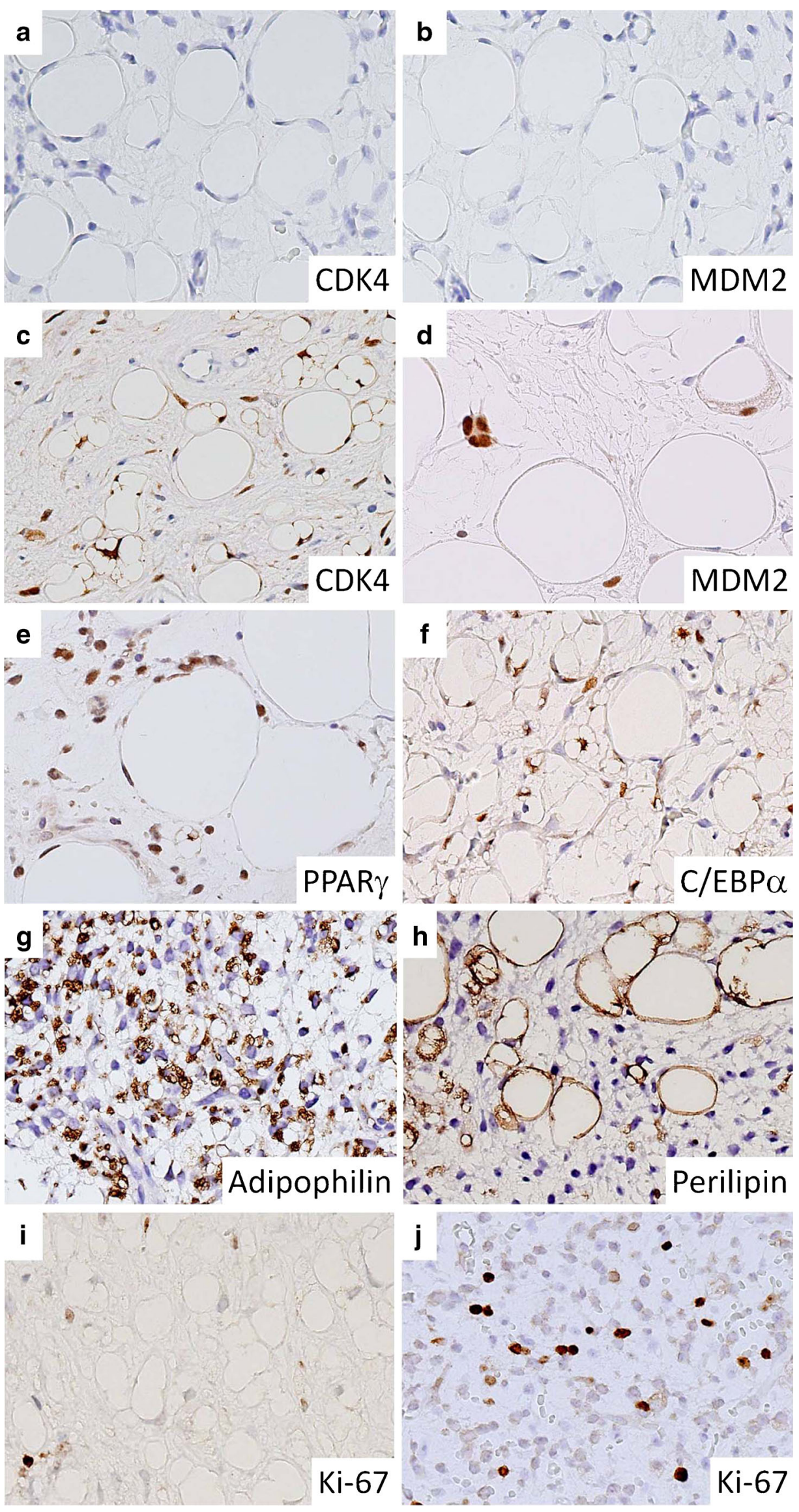


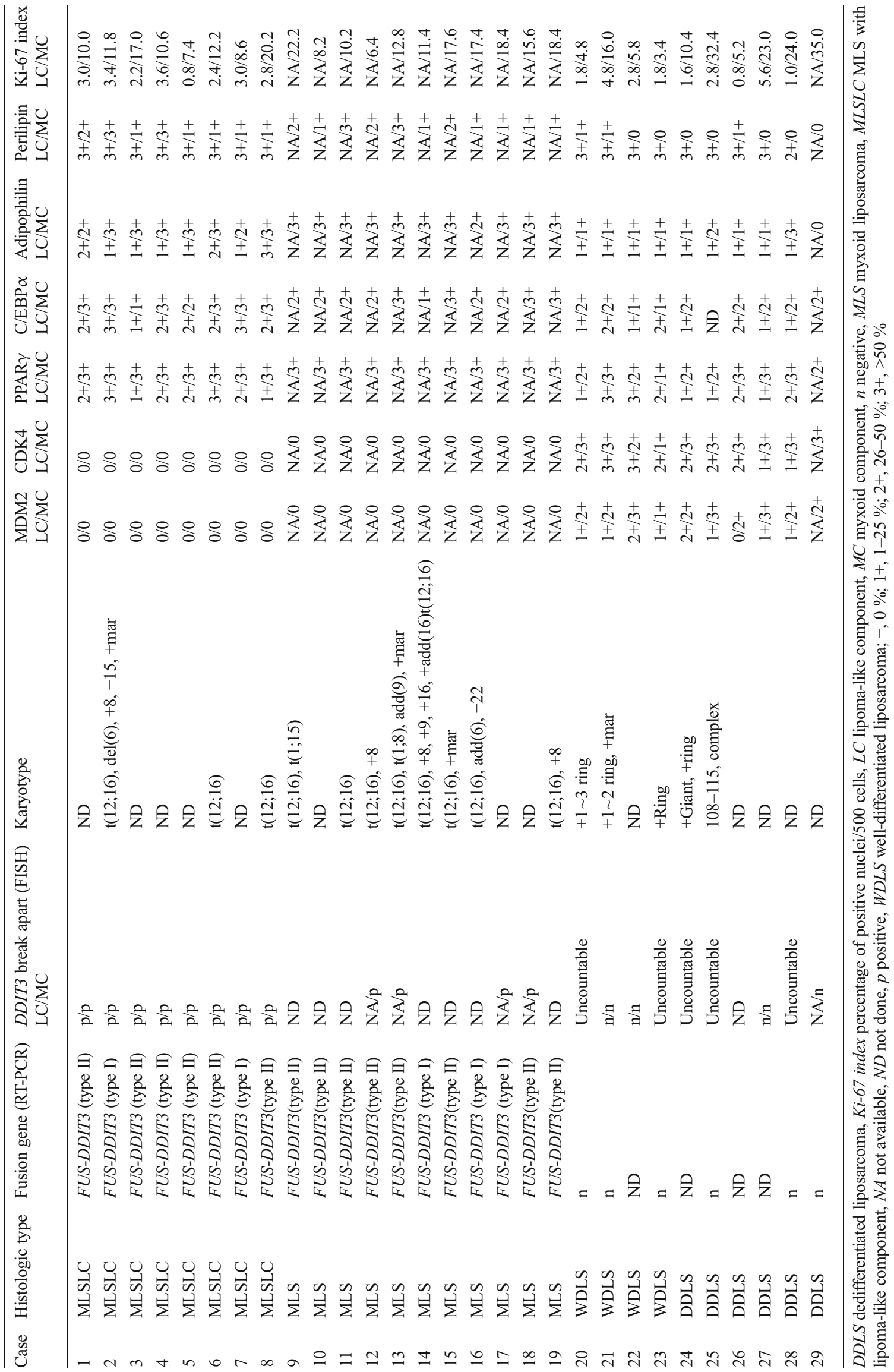


a

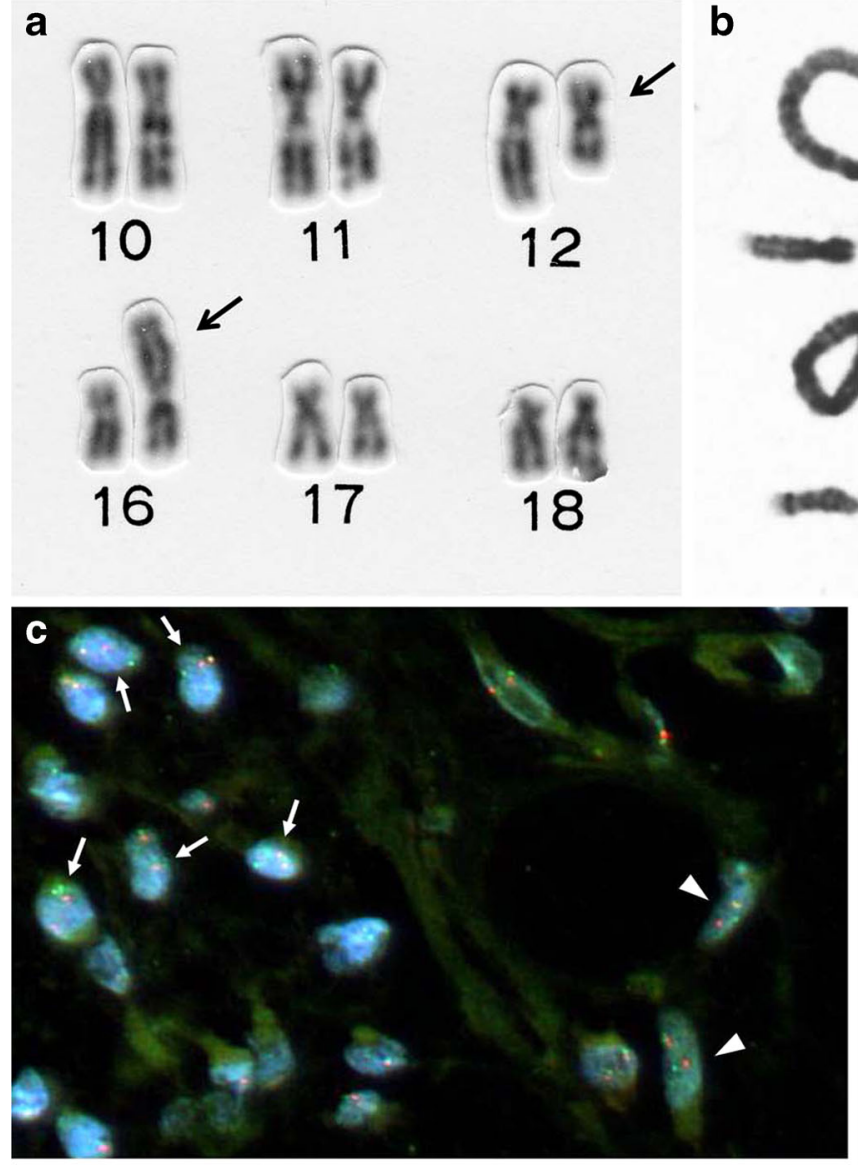

d

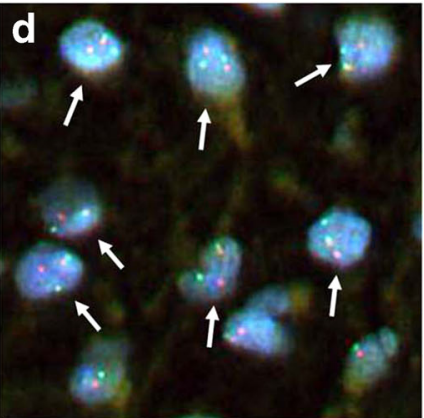

e MLSLC \begin{tabular}{l|l|l|l|} 
MLS & WD & DD & N
\end{tabular}

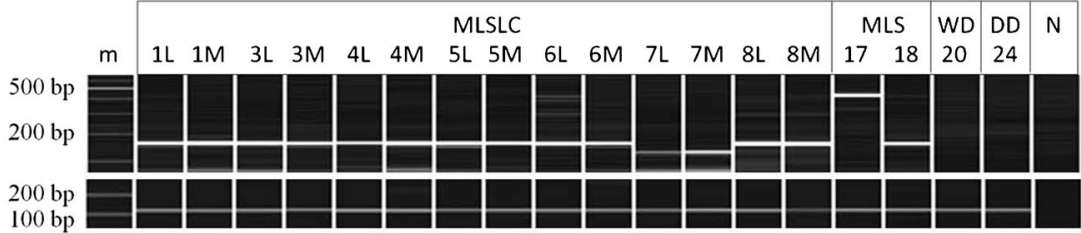

Fig. 4 Cytogenetic and molecular analysis of liposarcoma. a Partial karyotype of myxoid liposarcoma with lipoma-like changes (MLSLC) (case 2) showing $\mathrm{t}(12 ; 16)(\mathrm{q} 13 ; \mathrm{p} 11)$ (arrows indicate derivative chromosomes resulting from the reciprocal translocation). $\mathbf{b}$ Well-differentiated liposarcoma (case 21) exhibits characteristic supernumerary ring chromosomes. c Fluorescence in situ hybridization (FISH) detecting DDIT3 break apart in MLSLC (case 7). In a transitional zone between myxoid (left) and lipoma-like (right) areas, both immature round cells (arrows) and well-differentiated adipocytic cells (arrowheads) exhibit break-apart signals of DDIT3. d Myxoid area of MLSLC (case 8) presenting break-apart signals of DDIT3 in many sarcoma cells. e Detection of FUS-DDIT3 fusion transcript by RT-PCR

because of the morphologic similarities, although the absence of hyperchromatic cells in the adipocytic areas of MLSLC may be a useful diagnostic clue [7]. The recent advances of molecular cytogenetic techniques including FISH and RT-PCR provide powerful tools for resolving these problems, as shown in the present study.

Our study demonstrated that MLSLCs containing peculiar lipoma-like components actually represent MLS, which is different from either true mixed-type LS or WDLS/DDLS with with the FUS ex5 and DDIT3 primer set amplifying a 159-bp (FUSDDIT3 type II) fragment (cases 1 to $10,13,14$, and 16) or a 435-bp (FUS-DDIT3 type I) fragment (case 17) and the FUS ex7 and DDIT3 primer set amplifying a 128-bp (FUS-DDIT3 type I) fragment (case 7). In each MLSLC, FUS-DDIT3 fusion transcript was found in both lipomalike $(L)$ and myxoid $(M)$ areas. Ordinary MLSs (cases 17 and 18) were also positive for the specific FUS-DDIT3, whereas well-differentiated $(W D)$ and dedifferentiated $(D D)$ liposarcomas (cases 20 and 24) showed no fusion transcript. $m$, size marker, 100 -bp DNA ladder; $N$, negative control. In the lower lanes, the concomitant detection of $\beta_{2}$ microglobulin gene transcript $(120 \mathrm{bp})$ confirmed the presence of amplifiable RNA

myxoid changes, on the basis of molecular cytogenetic features. Both lipoma-like and myxoid components of the same MLSLC exhibited the identical abnormality, FUS-DDIT3 specific for MLS, which was confirmed by FISH and RT-PCR. There was a clear cut difference between MLSLC and WDLS/DDLS with myxoid changes; the former was positive for $\mathrm{t}(12 ; 16) / F U S-D D I T 3$ and negative for giant marker/ring chromosomes, whereas the latter exhibited converse characteristics. 
The occurrence of true mixed-type LS may be very rare, if it can exist as a distinct entity. Meis-Kindblom et al. [17] described a series of 30 cases of LS including a case of mixed LS, which is composed of WDLS and MLS with a coexistence of ring/giant marker chromosome (characteristic of WDLS) and $\mathrm{t}(12 ; 16) / F U S-D D I T 3$ (specific for MLS). Unfortunately, however, no FISH data from the WDLS component of the tumor was shown in this case. Mentzel et al. [27] reported another example of mixed-type LS consisting of ALT/WDLS and MLS components. By FISH analysis, they found amplifications of $M D M 2$ and $C D K 4$ genes in ALT/WDLS areas and translocations of DDIT3 and FUS genes in MLS areas, although their immunohistochemical study failed to demonstrate clear nuclear expressions of MDM2 and CDK4 in the tumor. On the other hand, Antonescu et al. [16] suggested that in many instances of mixed MLS+WDLS or translocationnegative MLS, the tumors may have represented predominantly myxoid WDLS or pleomorphic LS with myxoid changes, as supported by the cytogenetic data in the respective reports. They considered that the presence of microscopic foci of lipoma-like or sclerosing areas, characteristic of WDLS, constitutes sufficient histologic evidence to exclude the diagnosis of MLS, as supported by the consistent absence of DDIT3 or FUS genomic rearrangements in such tumors.

Recently, de Vreeze et al. [5] analyzed eight cases of LS designated as mixed-type LS with combined patterns of WDLS and MLS. By immunohistochemical and molecular data, they concluded that these mixed-type LSs should not be regarded as collision tumors, but as an extreme variant of morphological entity, explaining biological contradiction of mixed-type LS.

In the present study, FUS-DDIT3 fusion gene was found in distinct lipoma-like components as well as myxoid areas of MLSLC, suggesting that tumor cells of some true MLSs have a potential to differentiate into mature adipocytes producing a lipoma-like masses mimicking WDLS. Conversely, ring/giant marker chromosomes as well as immunohistochemical expressions of CDK4 and MDM2 characteristic of ALT/ WDLS were never found in any case of MLS with a lipomalike component.

Little is known about the mechanism of adipocytic differentiation in MLSLC and ordinary MLS, although various factors have been supposed to be concerned with the development of fat cells. Some molecular studies [13, 35] suggested that FUS-DDIT3 prevents the development of adipocytic precursors in MLS by repressing PPAR $\gamma$ and $\mathrm{C} / \mathrm{EBP} \alpha$. On the other hand, based on a comparative ultrastructural and RT-PCR analysis of MLS/RCLS, Huang and Antonescu [36] found that the variation of FUS-DDIT3 fusion transcript showed no apparent impact on adipogenesis of MLS, and they considered that other factors might be implicated in their level of differentiation.

Normal adipogenesis is thought to occur in two stages: commitment of mesenchymal stem cells to a preadipocyte fate, followed by terminal differentiation to mature adipocytes [29]. Adipogenic stimuli induce terminal differentiation in committed preadipocytes through the epigenomic activation of PPAR $\gamma$. The coordination of PPAR $\gamma$ with C/EBP transcription factors maintains adipocyte gene expression.

In our study, both PPAR $\gamma$ and $\mathrm{C} / \mathrm{EBP} \alpha$ were found in each type of LSs with or without mature lipoma-like components. The results are in accord with the molecular analysis by Tontonoz et al. [14]. Recent studies suggested that PPAR $\gamma$ may regulate the expression of lipid droplet-associated proteins including adipophilin and perilipin. Adipophilin associates with smaller neutral lipid storage droplets located within many tissues, whereas perilipin is located on the surface of larger triacylglycerol droplets in mature adipocytes and on cholesterol ester droplets in steroidogenic cells [30, 31]. In the present study, we found that adipophilin was strongly expressed in tiny fat droplets of immature lipoblastic cells of MLSLC and ordinary MLS, whereas mature adipocytes in lipoma-like component of MLSLC exhibited weak expression of this protein. On the other hand, perilipin showed a strong positive staining in large fat vacuoles of signet ring and multivacuolated lipoblasts as well as mature adipocytes in lipoma-like components of MLSLC and WDLS, but myxoid areas of MLSLC and DDLS contained a few cells possessing small fat vacuoles positive for perilipin. The combined immunohistochemical detection of adipophilin and perilipin may provide a useful ancillary tool for identification of lipoblastic cells in soft tissue sarcomas, since the former is localized in less-differentiated lipoblasts, and the latter is confined to more mature lipoblasts and fat cells.

Immunostaining for Ki-67 (MIB-1) demonstrated a lower labeling index of the nuclei in lipoma-like components of MLSLC, when compared with myxoid areas of the same tumor as well as ordinary MLS $(p<0.001)$. The results suggest that well-differentiated tumor cells resembling mature adipocytes in the lipoma-like components possess a lower level of proliferative activity, whereas undifferentiated or poorly differentiated cells in the myxoid areas of MLS with or without lipoma-like components retain high proliferative activities.

The clinical behavior of LS is highly dependent on the histologic subtype and the location of tumor $[1,2,4,17$, 25]. The prognosis of MLS involving the deep soft tissue of the extremities is generally favorable when appropriately treated by a complete wide surgical excision with or without a combined radiation or chemotherapy. In the present study, one $(20 \%)$ of five MLSLCs followed up had a recurrent tumor but no metastases, whereas four (44\%) of nine ordinary MLSs showed local recurrences and two (22\%) of these patients suffered from remote metastases. It cannot be denied that MLSLC may be less aggressive in clinical behavior when compared with ordinary MLS, although no appropriate statistical analysis could be applied in the present study because of the insufficient numbers of the patients. A large-scale study 
with sufficient numbers of patients is required to clarify the biological behavior of MLSLC. On the other hand, the overall poor prognosis of retroperitoneal WDLS may result from the frequent occurrence of dedifferentiation, in addition to late tumor detection, involvement of vital structures, and inability to achieve complete resection $[1,2,25]$.

In conclusion, our molecular cytogenetic study enabled the distinction between MLSLC and WDLS/DDLS with myxoid changes. The recognition of these peculiar conditions is important, because there are considerable differences of clinical behavior and prognosis between these distinct sarcomas presenting similar morphologic features. In addition, the combined immunohistochemical detection of adipophilin and perilipin may provide a useful ancillary tool for identification of lipoblastic cells in soft tissue sarcomas, since the former is localized in less-differentiated lipoblasts, and the latter is chiefly confined to more mature lipoblasts and fat cells.

Acknowledgments This work was supported in part by the Japan Society for the Promotion of Science (JSPS) KAKENHI Grant (No. 23590419)

Conflict of interest The authors declare no conflict of interest.

Open Access This article is distributed under the terms of the Creative Commons Attribution License which permits any use, distribution, and reproduction in any medium, provided the original author(s) and the source are credited.

\section{References}

1. Fletcher CDM, Bridge JA, Hogendoorn P, Mertens F (2013) WHO classification of tumours of soft tissue and bone, 4 th edn. IARC press, Lyon

2. Goldblum JR, Folpe AL, Weiss SW (2014) Enzinger and Weiss's soft tissue tumors, 6th edn. Mosby, Elsevier, Philadelphia

3. Miettinen M (2010) Modern soft tissue pathology: tumors and nonneoplastic conditions. Cambridge University Press, Cambridge

4. Boland JM, Colby TV, Folpe AL (2012) Liposarcomas of the mediastinum and thorax: a clinicopathologic and molecular cytogenetic study of 24 cases, emphasizing unusual and diverse histologic features. Am J Surg Pathol 36(9):1395-1403. doi:10.1097/PAS. 0b013e3182562bc1

5. de Vreeze RS, de Jong D, Koops W, Nederlof PM, Ariaens A, Haas RL, van Coevorden F (2011) Oncogenesis and classification of mixed-type liposarcoma: a radiological, histopathological and molecular biological analysis. Int J Cancer 128(4):778-786. doi:10.1002/ ijc. 25390

6. Nakanishi H, Araki N, Joyama S, Higuchi C, Mano M, Ishiguro S, Itoh K, Ueda T, Yoshikawa H (2004) Myxoid liposarcoma with adipocytic maturation: detection of TLS/CHOP fusion gene transcript. Diagn Mol Pathol 13(2):92-96

7. Sioletic S, Dal Cin P, Fletcher CD, Hornick JL (2013) Welldifferentiated and dedifferentiated liposarcomas with prominent myxoid stroma: analysis of 56 cases. Histopathology 62(2):287293. doi:10.1111/j.1365-2559.2012.04348.x

8. Turc-Carel C, Limon J, Dal Cin P, Rao U, Karakousis C, Sandberg AA (1986) Cytogenetic studies of adipose tissue tumors. II.
Recurrent reciprocal translocation $\mathrm{t}(12 ; 16)(\mathrm{q} 13 ; \mathrm{p} 11)$ in myxoid liposarcomas. Cancer Genet Cytogenet 23(4):291-299

9. Ohjimi Y, Iwasaki H, Ishiguro M, Ohgami A, Yoshitake K, Fujita C, Kikuchi M, Shinohara N, Kaneko Y (1992) Myxoid liposarcoma with $t(12 ; 16)(q 13 ; p$ 11). Possible usefulness of chromosome analysis in a poorly differentiated sarcoma. Pathol Res Pract 188(6):736741

10. Ohjimi Y, Iwasaki H, Kaneko Y, Ishiguro M, Ohgami A, Fujita C, Shinohara N, Yoshitake K, Kikuchi M (1992) Chromosome abnormalities in liposarcomas. Cancer Genet Cytogenet 64(2):111-117

11. Panagopoulos I, Mandahl N, Ron D, Hoglund M, Nilbert M, Mertens F, Mitelman F, Aman P (1994) Characterization of the CHOP breakpoints and fusion transcripts in myxoid liposarcomas with the 12;16 translocation. Cancer Res 54(24):6500-6503

12. Tallini G, Akerman M, Dal Cin P, De Wever I, Fletcher CD, Mandahl N, Mertens F, Mitelman F, Rosai J, Rydholm A, Sciot R, Van den Berghe H, Van den Ven W, Vanni R, Willen H (1996) Combined morphologic and karyotypic study of 28 myxoid liposarcomas. Implications for a revised morphologic typing, (a report from the CHAMP Group). Am J Surg Pathol 20(9):1047-1055

13. Kuroda M, Ishida T, Takanashi M, Satoh M, Machinami R, Watanabe $\mathrm{T}$ (1997) Oncogenic transformation and inhibition of adipocytic conversion of preadipocytes by TLS/FUS-CHOP type II chimeric protein. Am J Pathol 151(3):735-744

14. Tontonoz P, Singer S, Forman BM, Sarraf P, Fletcher JA, Fletcher CD, Brun RP, Mueller E, Altiok S, Oppenheim H, Evans RM, Spiegelman BM (1997) Terminal differentiation of human liposarcoma cells induced by ligands for peroxisome proliferatoractivated receptor gamma and the retinoid $\mathrm{X}$ receptor. Proc Natl Acad Sci U S A 94(1):237-241

15. Hisaoka M, Tsuji S, Morimitsu Y, Hashimoto H, Shimajiri S, Komiya S, Ushijima M (1998) Detection of TLS/FUS-CHOP fusion transcripts in myxoid and round cell liposarcomas by nested reverse transcription-polymerase chain reaction using archival paraffinembedded tissues. Diagn Mol Pathol 7(2):96-101

16. Antonescu CR, Elahi A, Humphrey M, Lui MY, Healey JH, Brennan MF, Woodruff JM, Jhanwar SC, Ladanyi M (2000) Specificity of TLS-CHOP rearrangement for classic myxoid/round cell liposarcoma: absence in predominantly myxoid well-differentiated liposarcomas. J Mol Diagn 2(3):132-138

17. Meis-Kindblom JM, Sjogren H, Kindblom LG, Peydro-Mellquist A, Roijer E, Aman P, Stenman G (2001) Cytogenetic and molecular genetic analyses of liposarcoma and its soft tissue simulators: recognition of new variants and differential diagnosis. Virchows Arch 439(2):141-151

18. Panagopoulos I, Lassen C, Isaksson M, Mitelman F, Mandahl N, Aman P (1997) Characteristic sequence motifs at the breakpoints of the hybrid genes FUS/CHOP, EWS/CHOP and FUS/ERG in myxoid liposarcoma and acute myeloid leukemia. Oncogene 15(11):13571362. doi:10.1038/sj.onc. 1201281

19. Iwasaki H, Nabeshima K, Nishio J, Jimi S, Aoki M, Koga K, Hamasaki M, Hayashi H, Mogi A (2009) Pathology of soft-tissue tumors: daily diagnosis, molecular cytogenetics and experimental approach. Pathol Int 59(8):501-521. doi:10.1111/j.1440-1827.2009.02401.x

20. Iwasaki H, Ohjimi Y, Ishiguro M, Isayama T, Fujita C, Kaneko Y, Kikuchi M, Shinohara N (1998) Supernumerary ring chromosomes and nuclear blebs in some low-grade malignant soft tissue tumours: atypical lipomatous tumours and dermatofibrosarcoma protuberans. Virchows Arch 432(6):521-528

21. Nishio J, Aoki M, Nabeshima K, Iwasaki H, Naito M (2012) Cytogenetic and molecular cytogenetic findings in giant dedifferentiated liposarcoma of the thigh. Oncol Rep 27(3):764 768. doi:10.3892/or.2011.1584

22. Nishio J, Iwasaki H, Ishiguro M, Ohjimi Y, Fujita C, Ikegami H, Ariyoshi A, Naito M, Kaneko Y, Kikuchi M (2003) Establishment of a novel human dedifferentiated liposarcoma cell line, FU-DDLS- 
1: conventional and molecular cytogenetic characterization. Int $\mathrm{J}$ Oncol 22(3):535-542

23. Sandberg AA (2004) Updates on the cytogenetics and molecular genetics of bone and soft tissue tumors: liposarcoma. Cancer Genet Cytogenet 155(1):1-24. doi:10.1016/j.cancergencyto.2004.08.005

24. de Vreeze RS, de Jong D, Tielen IH, Ruijter HJ, Nederlof PM, Haas RL, van Coevorden F (2009) Primary retroperitoneal myxoid/round cell liposarcoma is a nonexisting disease: an immunohistochemical and molecular biological analysis. Mod Pathol 22(2):223-231. doi: 10.1038/modpathol.2008.164

25. Evans HL (2007) Atypical lipomatous tumor, its variants, and its combined forms: a study of 61 cases, with a minimum follow-up of 10 years. Am J Surg Pathol 31(1):1-14. doi:10.1097/01.pas. $0000213406.95440 .7 \mathrm{a}$

26. Hisaoka M, Morimitsu Y, Hashimoto H, Ishida T, Mukai H, Satoh H, Motoi T, Machinami R (1999) Retroperitoneal liposarcoma with combined well-differentiated and myxoid malignant fibrous histiocytoma-like myxoid areas. Am J Surg Pathol 23(12):1480 1492

27. Mentzel T, Palmedo G, Hantschke M, Woziwodzki J, Beck C (2008) Mixed-type liposarcoma: clinicopathological, immunohistochemical, and molecular analysis of a case arising in deep soft tissues of the lower extremity. Virchows Arch 453(2):197-201. doi:10.1007/ s00428-008-0624-7

28. Deyrup AT, Chibon F, Guillou L, Lagarde P, Coindre JM, Weiss SW (2013) Fibrosarcoma-like lipomatous neoplasm: a reappraisal of socalled spindle cell liposarcoma defining a unique lipomatous tumor unrelated to other liposarcomas. Am J Surg Pathol 37(9):1373-1378. doi:10.1097/PAS.0b013e31829562ed

29. Cristancho AG, Lazar MA (2011) Forming functional fat: a growing understanding of adipocyte differentiation. Nat Rev Mol Cell Biol 12(11):722-734. doi:10.1038/nrm3198
30. Bildirici I, Roh CR, Schaiff WT, Lewkowski BM, Nelson DM, Sadovsky Y (2003) The lipid droplet-associated protein adipophilin is expressed in human trophoblasts and is regulated by peroxisomal proliferator-activated receptor-gamma/retinoid X receptor. J Clin Endocrinol Metab 88(12):6056-6062

31. Dalen KT, Schoonjans K, Ulven SM, Weedon-Fekjaer MS, Bentzen TG, Koutnikova H, Auwerx J, Nebb HI (2004) Adipose tissue expression of the lipid droplet-associating proteins S3-12 and perilipin is controlled by peroxisome proliferator-activated receptor-gamma. Diabetes 53(5):1243-1252

32. Shaffer LG, McGowan-Jordan J, Schmid M (2013) ISCN 2013: an international system for human cytogenetic nomenclature. Karger, Basel

33. Narendra S, Valente A, Tull J, Zhang S (2011) DDIT3 gene breakapart as a molecular marker for diagnosis of myxoid liposarcomaassay validation and clinical experience. Diagn Mol Pathol 20(4): 218-224. doi:10.1097/PDM.0b013e3182107eb9

34. Powers MP, Wang WL, Hernandez VS, Patel KS, Lev DC, Lazar AJ, Lopez-Terrada DH (2010) Detection of myxoid liposarcomaassociated FUS-DDIT3 rearrangement variants including a newly identified breakpoint using an optimized RT-PCR assay. Mod Pathol 23(10):1307-1315. doi:10.1038/modpathol.2010.118

35. Perez-Mancera PA, Bermejo-Rodriguez C, Sanchez-Martin M, AbolloJimenez F, Pintado B, Sanchez-Garcia I (2008) FUS-DDIT3 prevents the development of adipocytic precursors in liposarcoma by repressing PPARgamma and C/EBPalpha and activating eIF4E. PLoS One 3(7): e2569. doi:10.1371/journal.pone.0002569

36. Huang HY, Antonescu CR (2003) Molecular variability of TLSCHOP structure shows no significant impact on the level of adipogenesis: a comparative ultrastructural and RT-PCR analysis of 14 cases of myxoid/round cell liposarcomas. Ultrastruct Pathol 27(4): $217-226$ 